\title{
Effect of Process Conditions on Interface Properties during Ultrasonic Consolidation Process
}

\author{
Song ZHANG ${ }^{1, a}$, Li-Li ZHENG ${ }^{2, b,}$, , Hui ZHANG ${ }^{1, c}$ \\ ${ }^{1}$ Department of Engineering Physics, Tsinghua University, China, 100086 \\ ${ }^{2}$ School of Aerospace Engineering, Tsinghua University, China, 100086

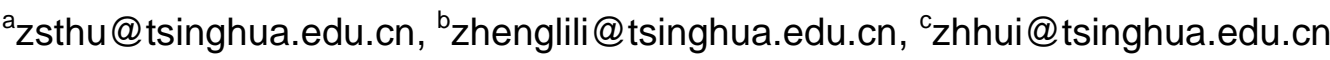 \\ ${ }^{*}$ Corresponding author
}

Keywords: Ultrasonic Consolidation, Interface Friction, Process Condition.

\begin{abstract}
A new friction model is built for ultrasonic consolidation process by considering deformation of foils due to the texture on the surface of sonotrode. Based on the friction model, a thermal-mechanical coupled finite elemental model is built to study the influence of process conditions on interface temperature and plastic strain. Study results show that the temperature distribution is mainly decided by the frictional heat generation at the contact interface and the plastic deformation mainly occurs at the top of a new foil where it is in contact with sonotrode. Also the applied load will mainly influence the interface plastic deformation and the interface temperature is mainly influenced by sonotrode oscillation amplitude. The sonotrode travel speed will mainly influence the oscillation time, thus influencing the interface temperature and plastic strain.
\end{abstract}

\section{Introduction}

Ultrasonic consolidation (UC) process is a solid state manufacturing process. During the UC process, metal foils are welded by ultrasonic oscillation of sonotrode. The temperature elevation due to frictional heating and stored plastic deformation are the main driving force for the bonding formation during UC process, which is believed to be caused by recrystallization at the contact area ${ }^{[1-3]}$. Much work has been done in order to study the heat transfer and plastic deformation in UC process. Schick and his coworkers ${ }^{[4]}$ built a finite elemental model to study the heat transfer and the thermal profiles during UC process. The results suggest that the voids and oxides at the foil interface will inhibit the thermal diffusion through the interface. Zhang and his coworkers ${ }^{[5]}$ has built a three dimensional model using the Coulomb's law of friction to describe the friction at the contact interface. Transient distributions and variables such as temperature and plastic deformation were analyzed and plastic deformation was proposed as a major phenomenon causing bond formation during UC process. Since frictional heating is considered the main heat source of UC process, it is of great importance to describe the friction phenomenon during the UC process. Schick and his coworkers ${ }^{[1]}$ conducted low-magnification optical observation of foil surface after UC process. They found it that the top surface of the foil was carved partially by sonotrode with some area unaffected during the UC process. Zhang and his coworkers ${ }^{[6]}$ have conducted tests to study the friction coefficient between Al 3003-H18 thin foils. The tests results show that the friction coefficient between Al 3003-H18 thin foils remains the same under different sliding speed but has a non-linear relationship with temperature.

Song and his coworkers ${ }^{[7]}$ has proposed the concept of "process map" in which the bonding quality is decided by the match of interface temperature elevation and plastic deformation. However, the influence of process conditions (applied load, sonotrode oscillaiton amplitude and sonotrode travel speed) on the interface parameters (interface temperature and plastic strain) still needs to be studied. In this paper, a friction model for the UC process is built based on the assumption that friction between layers occurs due to deformations of foil. And the influence of delay of sonotrode oscillation 
is also considered in the thermal-mechanical coupled finite elemental model based on the new friction model. Heat transfer and plastic deformation are studied in detail for the consolidation of $\mathrm{Al}$ alloy.

\section{Physical and Mathematic Model}

During the UC process, newly added foils are pressed by sonotrode onto the baseplate (or foils already welded). Frictional heating happens when the foil vibrates which is driven by the oscillating sonotrode (see Fig.1). During the current UC process, an applied load of $3 \mathrm{kN}$ to $10 \mathrm{kN}$ is always used and this also brings a very high frictional force between foil and baseplate (or foil). With the frictional heating at the interface, temperature near the interface is increased. Since plastic deformation also happens for material near the interface, recrystallization will occur at the interface, which will bring metallurgical bond between foils. The simplified conservation equations of thermal-mechanical coupled process can be expressed as ${ }^{[7]}$ :

$$
\begin{aligned}
& \frac{\partial \sigma_{i j}}{\partial x_{j}}+\rho b_{i}-\rho \frac{\partial^{2} u_{i}}{\partial t^{2}}=0 \\
& \rho c \frac{\partial T}{\partial t}=k \frac{\partial^{2} T}{\partial x_{i}^{2}}+\beta \sigma_{i j} \dot{\varepsilon}_{i j}^{p}
\end{aligned}
$$

where $\sigma_{i j}$ is the Cauchy stress tensor, $x_{i}$ is the space coordinate, $\rho$ is the density, $b_{i}$ is the body force per unit volume, $u_{i}$ is the displacement, $t$ is the time, $c$ is heat capacity, $T$ is temperature, $k$ is the heat conductivity, $\beta$ is the conversion fraction of plastic deformation work into heat and $\dot{\varepsilon}_{i j}^{p}$ is the plastic strain rate. In the energy conservation equation, the source term $\beta \sigma_{i j} \dot{\varepsilon}_{i j}^{p}$ is the heat converted from plastic deformation work and a conversion fraction of $\beta=0.3^{[8]}$ is used.

Heat is generated by friction at the interface between foils, and it will dissipate into the adjacent bulk materials by conduction. The rate of heat generated by friction is calculated by:

$$
\dot{q}=\alpha \cdot(\mu p+b) \cdot v
$$

where $\alpha$ is the fraction of frictional work converted into heat, $v$ is sliding speed, $\mu$ is the coefficient of friction, $p$ is the contact normal force and $b$ is the contact cohesion.

Eq. 3 shows that the frictional heat generation is mainly proportional the normal cantact stress and the average velocity. The sonotrode driven by the transducer move as a sine function of time with a fixed frequency of $f$. The displacement in " $z$ " direction can be expressed with the function of $u_{z}=A \sin 2 \pi f t$, where $A$ is the amplitude of oscillation. Also the velocity in " $z$ " direction can be obtained by first order derivation of displacement as $v_{z}=2 \pi f A \cos 2 \pi f t$. The average velocity in the direction of oscillation is proportional to the oscillation amplitude which decides the relative displacement of friction.

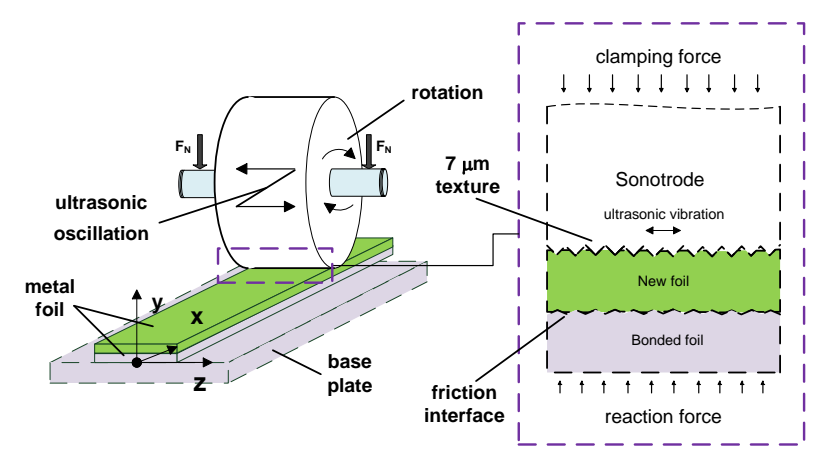

Fig.1 Schematic of UC Machine System and Contact Conditions for Different Interfaces 

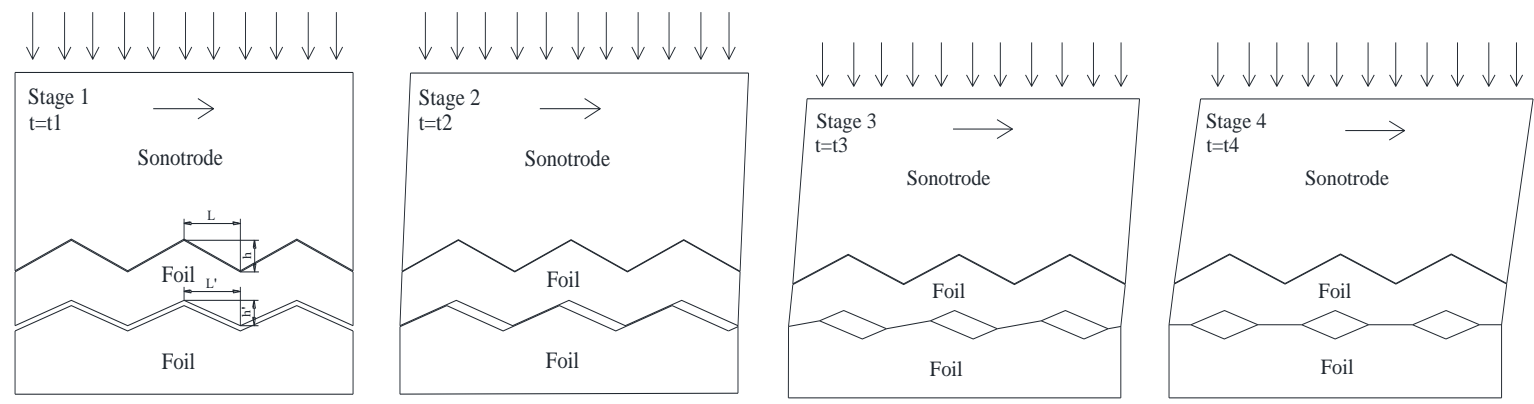

Fig.2 Schematic of Delay of Sonotrode Oscillation when Breaking the Friction

Figure 2 shows the delay of the sonotrode oscillation at the interface between sonotrode and foil when breaking the friction between foils. When the sonotrode is pressed onto the newly added foils (stage 1 in Fig.2), top surface of newly added foil will deform due to the texture on the contacting surface of sonotrode. At the same time, the bottom surface of newly added foil will also deform due to texture on the surface of bonded foil which is generated during the former run of consolidaiton. Since the deformation at the interface between newly added foil and bonded foil is little than that between sonotrode and foil, friction at the former interface will be broken first. When the sonotrode moves from time $t_{1}$ to $t_{2}$, the resulting shear stress at the foil interface is not enough to break the lock, so there is no displacement for the bottom surface of the new foil. After $t_{2}$, the lock at the foil interface began to be broken with the increase of sonotrode movement until time $t_{4}$. This causes a delay of oscillation at the interface between foils. Since the deformation height $h^{\prime}$ will increase with the applied load, the delay of oscillation will also increase. A delay factor is introduced to represent the magnitude of sonotrode oscillation delay based on the assumption that the oscillation amplitude at the foil interface decreases linearly with the applied load.

In order to simplify the numerical model, no rotation is considered in the thermal-mechanical model and the computation time is decided by an equivalent frictional heat generation. Eq. 3 show that the frictional heat flux is proportional to the normal stress at the contact interface. When no rotation considered, the equivalent computation time should be smaller than the real time during which a given point is in contact with sonotrode. A equivalent computation time is used by controlling the equivalent friction heat generation. The equivalent computation time can be expressed as:

$$
t_{c}=\frac{\int_{0}^{+\infty} \sigma_{N} \mathrm{~d} x}{\sigma_{x=0} \cdot v_{x}}
$$

where, $\sigma_{\mathrm{x}}$ is the normal stress distribution in the " $x$ " direction, $\sigma_{\mathrm{x}=0}$ is the normal stress where $x=0, v_{\mathrm{x}}$ is the travel speed of sonotrode.

\section{Results and Discussion}

A finite elemental model is built with the ANSYS software as described in the former paper about "process map"[7] . A fully coupled thermal - mechanical simulation is conducted without considering the rotation of sonotrode. Based on the analysis of sonotrode oscillation delay, new oscillation amplitude under different applied load is used. Also the simulation time under different process conditions is also recalculated based on the analysis of equivalent frictional energy generation. Figure 3 shows the computational domain and meshes in the new model. Since the first layer is welded onto the baseplate using the same optimized operating conditions for different cases, the welding of the second layer is simulated. 

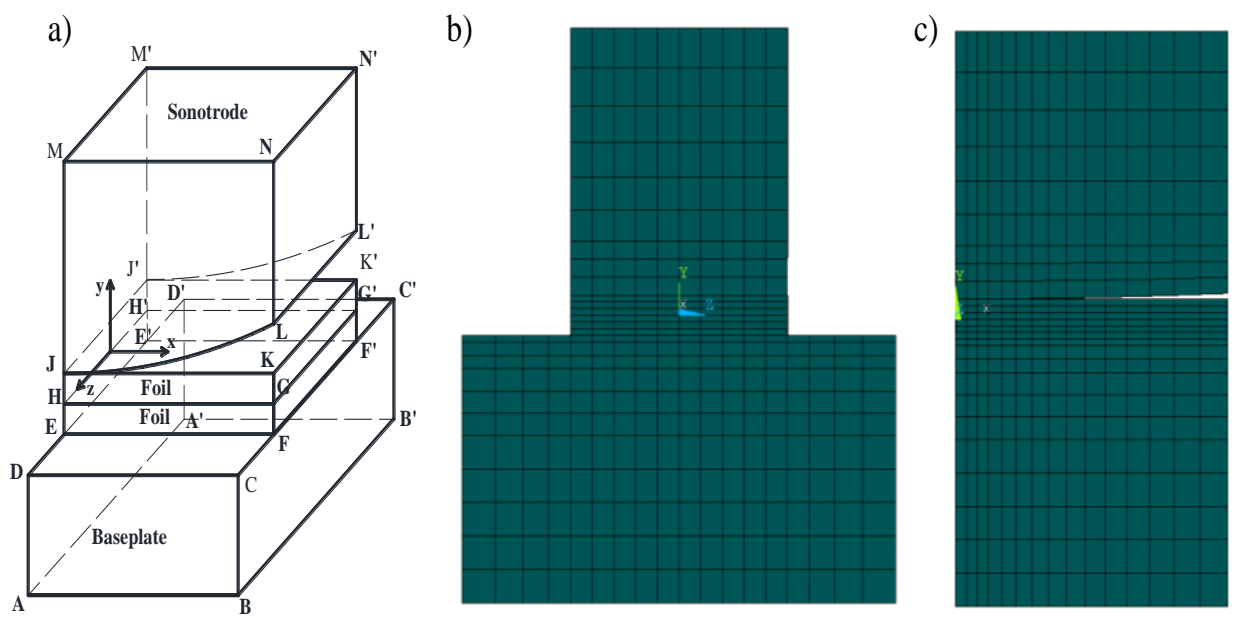

Fig.3 Computational Domain and Meshes Used in the Numerical Model, a) Computational Domain, b) Meshes in $y$ - $z$ Plane, c) Meshes in $x-y$ Plane

a)

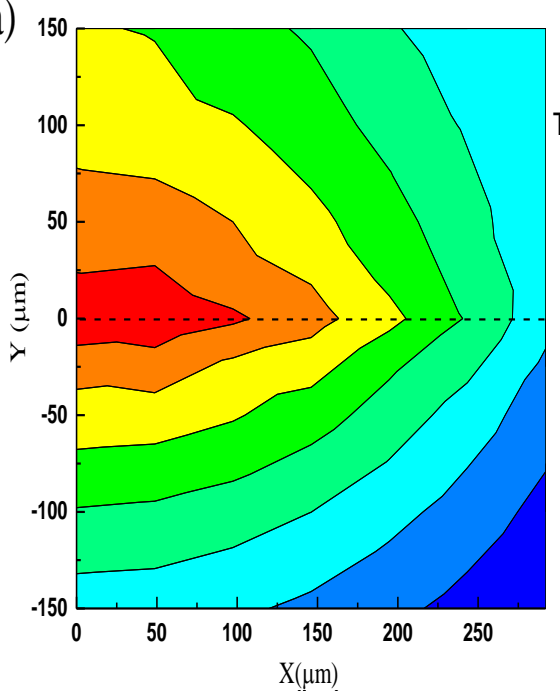

b)

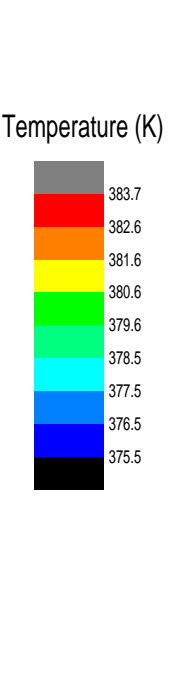

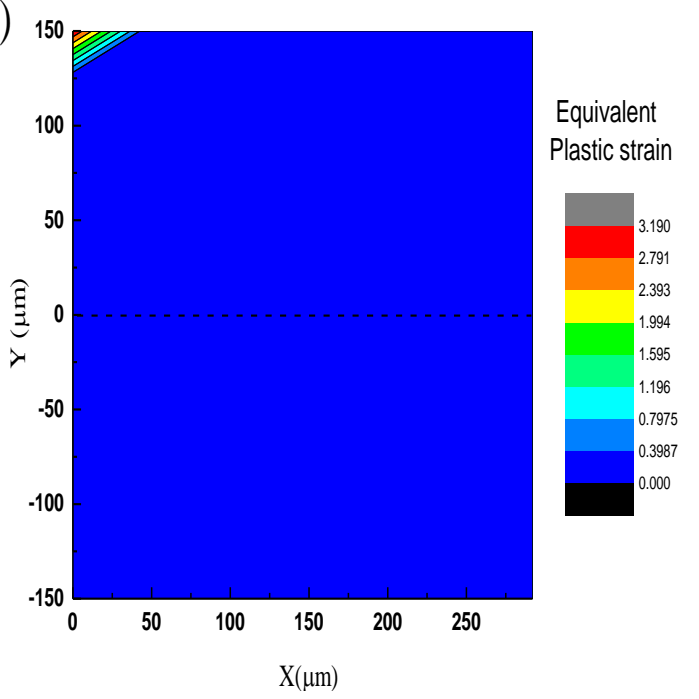

Fig.4 Temperature and Equivalent Plastic Strain Distribution at $z=0$, a) Temperature Distribution, $b$ ) equivalent Plastic Strain Distribution

A baseline case is chosen to study the temperature and plastic deformation during the UC process. For the baseline case the preheating temperature is $370 \mathrm{~K}$, applied load is $5500 \mathrm{~N}$, sonotrode travelling speed is $63.5 \mathrm{~mm} / \mathrm{s}$, oscillation amplitude is $21 \mu \mathrm{m}$ and an oscillation delay factor of $4 \%$ per $1 \mathrm{kN}$ is used. Figure 4 shows the temperature and equivalent plastic strain distribution at $z=0$ when the consolidaiton process is finished. The temperature distribution in Fig.4a shows that the highest temperature occurrs at the contact interface region where the fictional heating happens. The equivalent plastic strain distribution in Fig.4b shows the largest deformation occurrs where the sonotrode is in contact with the foil. This phenomenon is supported by microstructure observations which shows that recrystallization happens mainly within $20 \mu \mathrm{m}$ below the contact interface ${ }^{[9]}$. This is because no more severe plastic deformation occurs at the contact interface between foils during the UC process, and recrystallization happens mainly due to the frictional heating effect at the contact interface and the plastic deformation generated during condolidaiton of last layer. The temperature at the origin point and the equivalent plastic strain where $x=z=0$ and $y=150 \mu \mathrm{m}$ are the maximum value of temperature and equivalent plastic strain at a given time, and they are selected to monitor the evolution of temperature and equivalent plastic stain. 

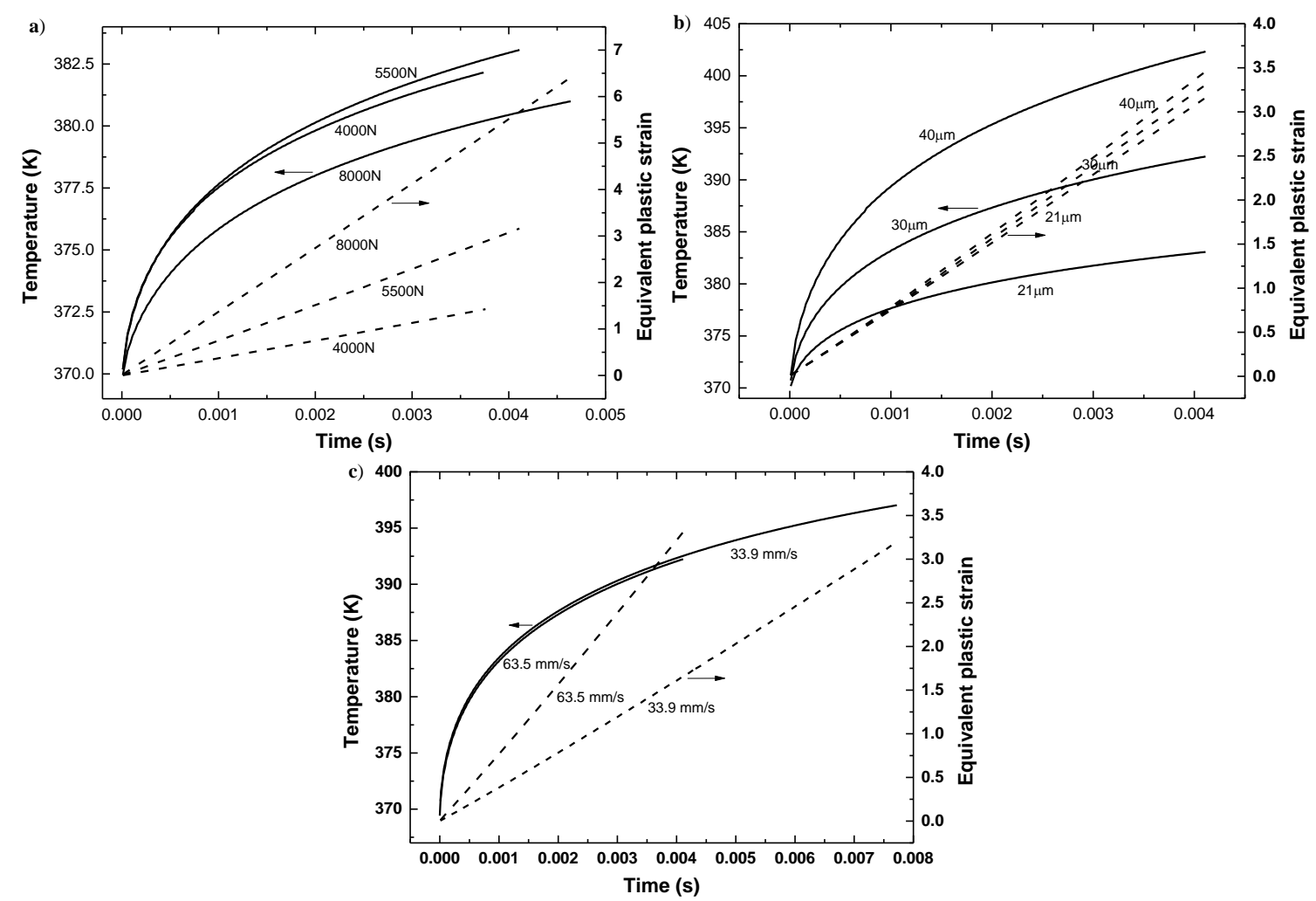

Fig.5 Temperature and Equivalent Plastic Strain Evolution as a Function of Time Under Different a) Applied Load, b) Amplitude, c) Speed

Based on the baseline case, cases with different process conditions are analyzed. Figure 5a shows the temperature and the equivalent plastic strain evolution under different applied load. It is shown that the highest temperature reached during UC process increases with applied load first and then begin to decrease. However the highest equivalent plastic strain reached will increase quickly with applied load. Figure $5 \mathrm{~b}$ shows the temperature and the equivalent plastic strain evolution under different amplitude. It is shown that the highest temperature reached increases quickly with the amplitude while highest equivalent plastic strain increases slowly with amplitude. This is because the increase of amplitude will increase the average velocity during UC process, thus increasing the frictional heat generation. With the increase of interface temperature, the equivalent plastic strain will also increase. Figure $5 \mathrm{c}$ shows the temperature and the equivalent plastic strain evolution under different speed. A lower sonotrode travel speed will increase the vibration time, thus increasing the interface temperature. However, there is no large change for the plastic strain since the friction conditions remain almost the same.

\section{Summary}

A friction model is built by considering the deformation of foils due to the texture on sonotrode. In the friction model, the sonotrode is considered to be locked to the top foil and friction only occurs at the contact interface of foils. Also there is a delay of sonotrode oscillation during the breaking of the lock between foils due to deformation. Based on the friction model and the delay effect, the influence of applied load, sonotrode oscillation amplitude and sonotrode travel speed on the interface properties is studied with a new thermal-mechanical coupled model. It is found that the temperature distribution is mainly decided by the frictional heat generation at the contact interface and the plastic deformation mainly happens at the top of a new foil where it is in contact with sonotrode. The applied load will mainly influence the interface plastic deformation and the interface temperature is mainly influenced by sonotrode oscillation amplitude. The sonotrode travel speed will mainly influence the oscillation time, thus influencing the interface temperature and plastic strain. 


\section{Acknowledgement}

The authors acknowledge the ultrasonic consolidation project sponsored by Tsinghua-Boeing joint research program.

\section{References}

[1] D.E. Schick, R.M. Hahnlen, R. Dehoff, P. Collins, S.S. Babu, M.J. Dapino, J.C. Lippold, Microstructural Characterization of Bonding Interfaces in Aluminum 3003 Blocks Fabricated by Ultrasonic Additive Manufacturing, Welding Journal. 89 (2010) 105s-115s.

[2] M.R. Sriraman, S.S. Babu, M. Short, Bonding characteristics during very high power ultrasonic additive manufacturing of copper, Scripta Materialia. 62 (2010) 560-563.

[3] E. Mariani, E. Ghassemieh, Microstructure evolution of $6061 \mathrm{O}$ Al alloy during ultrasonic consolidation: an insight from electron backscatter diffraction, Acta Materialia. 58 (2010) 2492-2503.

[4] D. Schick, S.S. Babu, D.R. Foster, M. Dapino, M. Short, J.C. Lippold, Transient thermal response in ultrasonic additive manufacturing of aluminum 3003, Rapid Prototyping Journal. 17 (2011) 369-379.

[5] C. Zhang, L.J. Li, A Coupled Thermal-Mechanical Analysis of Ultrasonic Bonding Mechanism, Metallurgical and Materials Transactions B-Process Metallurgy and Materials Processing Science. 40 (2009) 196-207.

[6] C. Zhang, X. Zhu, L. Li, A study of friction behavior in ultrasonic welding (consolidation) of aluminum, 87th FABTECH International and AWS Welding Show Professional Program. (2006).

[7] S. Zhang, D. Yi, H. Zhang, L. Zheng, Y. Zhang, Z. Yang, M. Norfolk, Towards understanding of ultrasonic consolidation process with "process map", Rapid Prototyping Journal. 21 (2015) 461-468.

[8] J. Hodowany, G. Ravichandran, A. Rosakis, P. Rosakis, Partition of plastic work into heat and stored energy in metals, Experimental Mechanics. 40 (2000) 113-123.

[9] M.R. Sriraman, M. Gonser, D. Foster, H. Fujii, S.S. Babu, M. Bloss, Thermal Transients During Processing of 3003 Al-H18 Multilayer Build by Very High-Power Ultrasonic Additive Manufacturing, Metall and Materi Trans B. 43 (2012) 133-144. 